\title{
Labour epidural practice in a tertiary training centre
}

\begin{abstract}
Dear Editor,
Labour analgesia provided through the central neuraxial approach is offered for parturients who are in their active stage of labour, usually after $2-4 \mathrm{~cm}$ of cervical dilatation. Lumbar epidural, the gold standard for labour analgesia, is recently recommended over other alternative methods of analgesia such as pressurised gaseous nitrous oxide, to minimise aerosol-related risks to healthcare teams. ${ }^{1}$ The labour epidural procedure requires advanced technical regional anaesthetic skills. It is uniquely associated with immense pressure to achieve timesensitive procedural success, as patients vocalise the unbearability of pain while the next of kin are present in the delivery suite room. The difficulties encountered are the unstable or frequently shifting patient positioning that dynamically alters the access to the epidural and subarachnoid space; anatomical changes in pregnancy; exaggerated lumbar lordosis; and distended epidural blood vessels during frequent active contractions. ${ }^{2}$ Maternal obesity further compounds this, resulting in adverse outcomes. ${ }^{3}$
\end{abstract}

Opportunities for learning non-obstetric epidural analgesia have significantly reduced due to the introduction of surgeon-led infiltration-analgesic practices, and truncal plane blocks such as the erector spinae plane (ESP). ${ }^{4}$ The introduction of protocolbased enhanced recovery after surgery (ERAS) has limited the use of central neuraxial blockade to facilitate early ambulation. This has resulted in obstetric labour epidurals being the first epidural technique that the trainees tend to perform on patients.

To address these gaps, trainees receive structured simulation-based knowledge, skills and cognitive training before they are allowed to perform epidurals on parturients. ${ }^{5}$ These include proficiency in technical skills achieved on epidural part-task trainer mannequins, followed by apprenticeship model of clinical observation and guided actual hands-on experiences. Crisis management and interprofessional team-based simulations are introduced subsequently to master the attributes of situational awareness and enhanced closed-loop communications.

The existing system of supervision of trainees emulates the entrustable professional activity-based models, ${ }^{6,7}$ where they are stratified based on their individual ability to perform the epidural procedure with technical accuracy, identify clinical variations (obesity and scoliosis), recognise patterns of complications (accidental dural puncture and intravascular epidural misplacement) and to promptly escalate when clinical acute deteriorations (maternal hypotension and local anaesthetic toxicity) occur. There is limited data clarifying whether epidural procedures performed by trainees were as safe as those done by specialists. There were inconsistent practices on how an epidural procedure is offered for trainees to attempt. These included mere electronic medical review of body weight and body mass index (BMI), and not by a structured assessment of anticipated difficulty by clinical evaluation. Cohort-based data on the anatomical variations and reported difficulties between various ethnicities and nationalities were limited.

We report a retrospective cohort study (December 2017 to May 2018), with data accessed through the electronic health records (computerised patient support system [CPSS] 2, Integrated Health Information Systems, Singapore) at National University Hospital, Singapore (full review: NHG-DSRB 2018/00453). The study included the review of scanned records of labour epidural procedures, pain audits and incidents reported in the immediate peripartum period until discharge. The data was analysed by SPSS Statistics software version 24.0 (IBM Corp, Armonk, US).

A total of 912 patients who received central neuraxial blockade for labour analgesia were screened. After excluding those with incomplete documentation, data from 727 patients were analysed. Of the patients analysed, $639(87.9 \%)$ received combined spinal epidural analgesia, $87(12.0 \%)$ received plain epidural analgesia and in $1(0.1 \%)$, the technique was unreported.

Majority of patients $(41.4 \%)$ belonged to the overweight category (BMI mean 27.96, standard deviation 5.17) and epidural depth was found to moderately correlate $(\mathrm{r}=0.53)$ with $\mathrm{BMI}(3.3 \mathrm{~cm}$ in $\mathrm{BMI}<18$ to $6.5 \mathrm{~cm}$ in $\mathrm{BMI}>40$ ). The cohort included patients stratified based on their ethnicities and further by nationalities within the same ethnicities. The population distribution of the ethnicities included patients of Chinese origin (46\%), Malay (20\%), Indian $(17 \%)$, Caucasian (3\%) and other ethnicities (14\%). The proportion of nationalities varied among the different ethnicities (e.g. Chinese: 30\% expatriate, 70\% local; Indian: $63 \%$ expatriate, $37 \%$ local).

Majority of epidurals $(90 \%)$ in Caucasians were achieved in the first attempt compared to $75 \%$ for 
Table 1. Distribution of ethnicities, level of epidural difficulty and complications

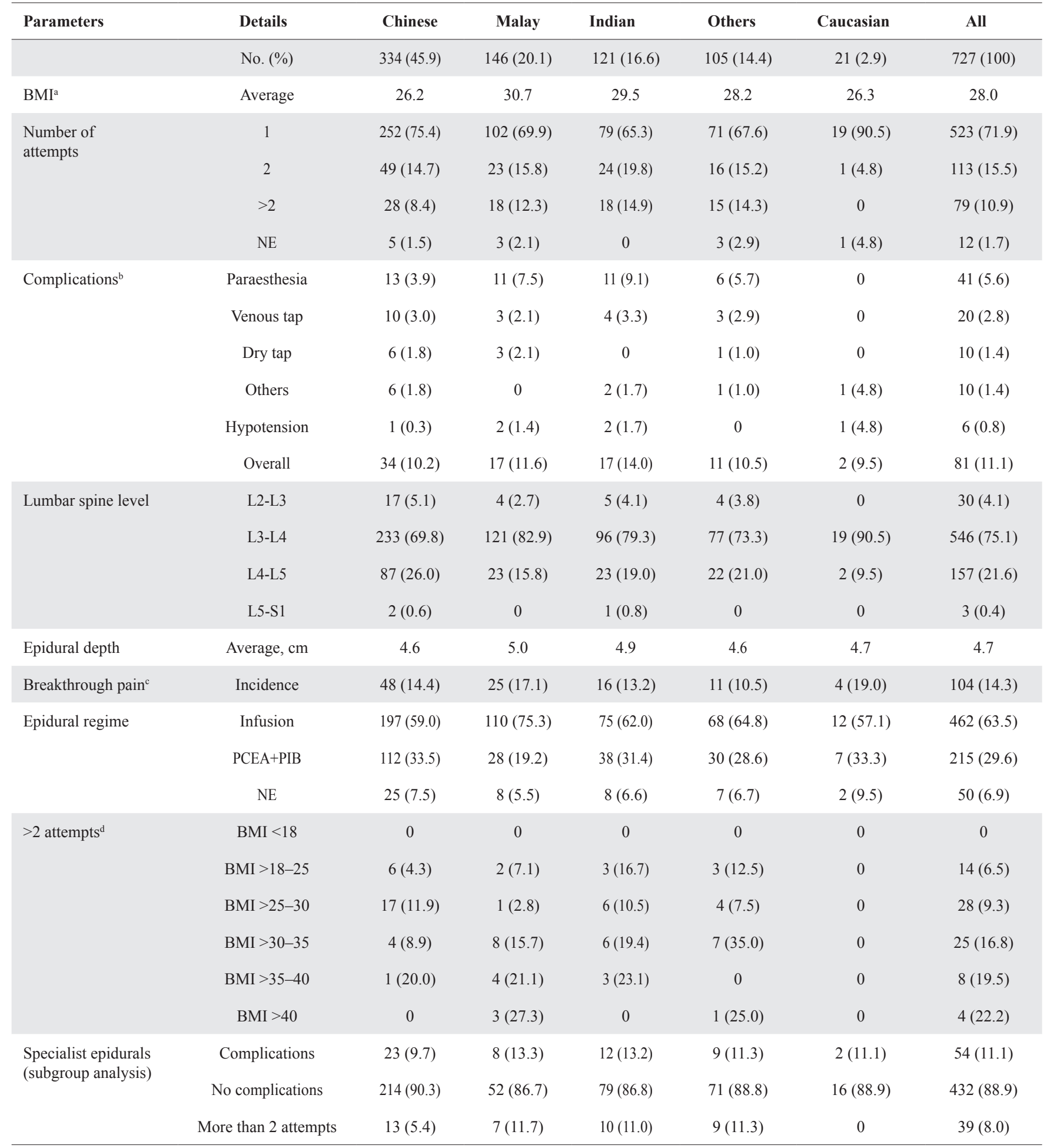

BMI: body mass index; NE: not entered or data unavailable; PCEA: patient-controlled epidural analgesia; PIB: programmed intermittent bolus

${ }^{a}$ BMI was calculated objectively by documented weight and height in electronic health records at the time of booking

${ }^{\mathrm{b}}$ Complications exclude breakthrough pain

${ }^{c}$ Epidural infusions had higher breakthrough pain (14.5\%) compared to PCEA+PIB (13.5\%)

${ }^{\mathrm{d}}$ Attempt is defined as number of reinsertions with new skin puncture and excludes the re-directions of needle during same insertion. Epidural depth can vary between the lumbar levels of same patient; an average is presented above. 
Chinese, $70 \%$ for Malay and $65 \%$ for Indian ethnicities. The rate of complications (residual neuropraxia, accidental dural puncture, etc.) was found to be highest in Malay ethnicity (25.7\%) followed by Indian (24.6\%), Caucasian $(23.8 \%)$, Chinese $(20.9 \%)$ and Others (19\%) (Table 1). Logistic regression analysis showed 3 times higher odds of incidence of complications $(P=0)$ if more than 2 attempts were needed for epidural placement.

Specialists with more than 5 years of experience performed $67 \%$ of epidurals. There were no significant differences in the overall rate of complications among the epidurals performed by specialists (11\%), trainees $(12 \%)$ and those performed by trainees that eventually required specialist support $(10 \%)$. This data reaffirmed the safety of existing structured training and supervision when labour epidurals are performed by trainees. Experienced midwives present in the procedure rooms are trained to summon for specialist presence when trainees struggle with technical complexities, providing parturients with an additional safety net from preventable harm.

Known predictors of anticipated epidural difficulty include grade or ease of palpation of spinous process, presence of truncal versus lateral obesity with BMI $>30$ and abdominal girth, degree of exaggerated lumbar lordosis, pre-procedural ultrasound documentations of spinal anatomy and abnormalities. ${ }^{8}$ The authors observed that besides BMI, technical difficulties result from variations of fat distribution that cause filling of "central valley" between the paraspinal muscles. These complexities that influence the level of difficulty in performing the procedure were not evident until the parturients were positioned to sit up for the epidural procedure. The distribution of adiposity did not relate to stratifications of patients by measurement of BMI. Factors that could be explored include body habitus pre-pregnancy, truncal or central obesity, and level of physical activity. ${ }^{9}$

Future prospective studies on comparison of rates of complication need to consider matching the level of expertise of proceduralists for the various ethnicities or when comparing the nationalities within the same ethnicities. ${ }^{10}$ Decisions for offering opportunities for trainees (on who does the epidural) in "difficult epidurals" should be made from direct assessment of surface anatomy or sono-visualisation (present spinal ultrasound rate is $<10 \%),{ }^{7}$ and by actual palpability of patients' lumbar spine. ${ }^{11}$ This "anticipated difficulty" should be evaluated and established in person by the specialist or experienced midwife, and not through remotely accessed electronic records suggesting calculated high or normal BMI based on height or weight. ${ }^{12}$ The study informs us of the safety of the existing structured training, with experienced nursing-supervision and escalation processes for specialist support in high-risk complex procedures, such as obstetric labour epidurals. The current study showed that the rates of complications and procedural concerns were comparable to global data on incidence of events (12.2\%) where proficiencybased training progression and validation of readiness of trainees were ascertained. ${ }^{13}$ The current system of structured introduction to complex clinical procedures, with guided specialist supervision for labour epidural analgesia and monitoring by experienced midwives, seems to be a safe practice and is commendable. Further prospective studies stratified by level of clinical expertise are needed to establish more concrete associations among anatomical variations, ethnicities and nationalities.

\section{Acknowledgements}

We are grateful for the support received from the obstetrics nurses and midwives of National University Hospital, Singapore. We appreciate the statistical support provided by Jolene Lee, medical student, Duke-NUS Medical School, Singapore.

\section{REFERENCES}

1. Ashokka B, Loh MH, Tan $\mathrm{CH}$, et al. Care of the Pregnant Woman with COVID-19 in Labor and Delivery: Anesthesia, Emergency cesarean delivery, Differential diagnosis in the acutely ill parturient, Care of the newborn, and Protection of the healthcare personnel. Am J Obstet Gynecol 2020;223:66-74.e3.

2. Paech MJ, Godkin R, Webster S. Complications of obstetric epidural analgesia and anaesthesia: a prospective analysis of 10,995 cases. Int J Obstet Anesth 1998; 7:5-11.

3. Loh HH, Taipin H, Said A. Maternal obesity and risk of adverse obstetric outcomes in Malaysia. Ann Acad Med Singap 2021; 50:285-8.

4. Saadawi M, Layera S, Aliste J, et al. Erector spinae plane block: A narrative review with systematic analysis of the evidence pertaining to clinical indications and alternative truncal blocks. J Clin Anesth 2021;68:110063.

5. Fuhrmann L, Østergaard HT, Østergaard D. Teaching epidural for labor analgesia. In: Capogna G (Ed). Epidural labor analgesia: Childbirth without pain. Springer, Cham; 2015, pp303-13.

6. Weller JM, Naik VN, San Diego RJ. Systematic review and narrative synthesis of competency-based medical education in anaesthesia. Br J Anaesth 2020;124:748-60.

7. Wisman-Zwarter $\mathrm{N}$, van der Schaaf $\mathrm{M}$, Ten Cate $\mathrm{O}$, et al. Transforming the learning outcomes of anaesthesiology training into entrustable professional activities: a Delphi study. Eur J Anaesthesiol 2016;33:559-67.

8. Borges BC, Wieczoreck P, Balki M, et al. Sonoanatomy of the lumbar spine of pregnant women at term. Reg Anesth Pain Med 2009;34:581-5. 
9. Weiniger CF, Cohen A, Aptekman B, et al. Prospective observational investigation of body habitus measurements and relationship to epidural depth in term pregnant women. Acta Anaesthesiol Scand 2020;64:677-84.

10. Elsharkawy H, Sonny A, Chin KJ. Localization of epidural space: A review of available technologies. J Anaesthesiol Clin Pharmacol 2017;33:16-27.

11. Rajagopalan S, Shah K, Guffey D, et al. Predictors of difficult epidural placement in pregnant women: A trainees' perspective. J Anaesthesiol Clin Pharmacol 2019;35:548-52.

12. Roystonn K, Abdin E, Sambasivam R, et al. Accuracy of selfreported height, weight and BMI in a multiethnic Asian population. Ann Acad Med Singap 2021;50:306-14.

13. Mohamed H, McAuliffe N, O'Connor R, et al. Proficiency-based progression training: implementing a novel approach to training for epidural analgesia in labour. Int J Obstet Anesth 2021;48:103213.
Balakrishnan Ashokka ${ }^{1,2} M H P E$, Deepika $\underline{\text { Arora }}^{3} M D$, Shrividya Niranjan Kumar ${ }^{4} M D$, Rachel Chin ${ }^{1}{ }^{M B B S}$, Rajesh $\underline{\text { Kannan }}{ }^{1}{ }_{F F A R C S I}$, Bryan ${ }_{\text {Ng }}{ }^{1}{ }_{F R C A}$, May-Han Loh ${ }^{1,2}$ MMed

${ }^{1}$ Department of Anaesthesia, National University Health System, Singapore

${ }^{2}$ National University of Singapore, Singapore

${ }^{3}$ Department of Anaesthesia, Newham Hospital, NHS Trust, London, UK

${ }^{4}$ Department of Anaesthesia, St Michael's Hospital, Ontario, Canada

Correspondence: Dr Balakrishnan Ashokka, Department of Anaesthesia, National University Hospital, Level 3 Main Building, 5 Lower Kent Ridge Road, Singapore 119074.

Email: ashokkab@gmail.com 